Communications in Applied Geometry.

Volume 1, Number 1 (2011) pp. 27-30

(C) Research India Publications

https://dx.doi.org/10.37622/CAG/1.1.2011.27-30

\title{
A Proof of the Poincaré Conjecture
}

\author{
Euich Miztani
}

\begin{abstract}
If it is possible to construct $M^{3}$ by gluing two boundaries of $S^{2}$ together, the Poincare conjecture will be true as well as other lower dimensional cases of $S^{1}$ and $S^{2}$ that are self-evident: This is the simplest proof. However, it has been considered impossible to glue them together directly unlike the cases $S^{1}$ and $S^{2}$, so that we have researched if the conjecture is true by different approaches. However, such a direct proof method will be performed by introducing an especial coordinate. In this paper, we discuss how to verify it with a direct method.
\end{abstract}

Keywords: reversed coordinate, three-manifold, three-sphere, simple connectivity.

2000 Mathematics Subject Classification: 57M40.

\section{A Reversed Coordinates}

First of all, let us introduce so to say a reversed coordinate. As shown in Figure 1, the coordinate's origin is located at infinite distance; positions of the origin and directions of the axes are reversed if comparing with the ordinary coordinate as shown in Figure 2 .

To know characteristics of the reversed coordinate, let us draw a graph in the reversed coordinate. For example, a graph of locations of the earth and the sun in the universe in the ordinary coordinate is drawn as shown in Figure 3. However, another drawing in the reversed coordinate is quite different as shown in Figure 4: The origin(; Earth) is drawn as a ring, the sun is surrounded by the earth, and the end of the universe is drawn as a point. 


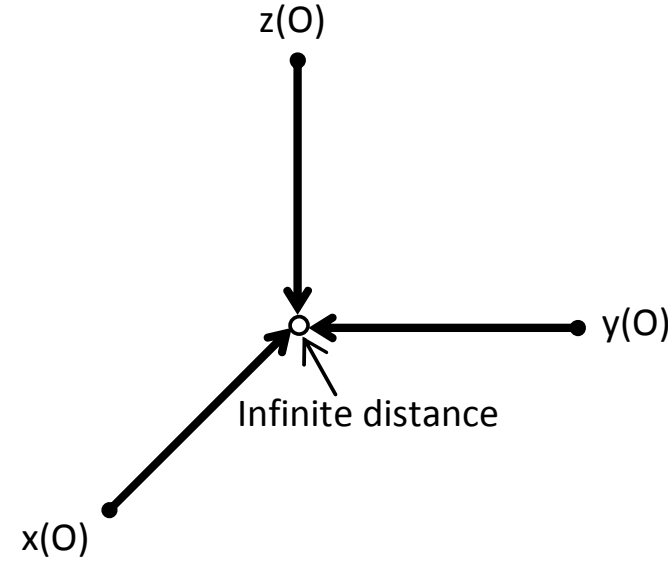

Figure 1

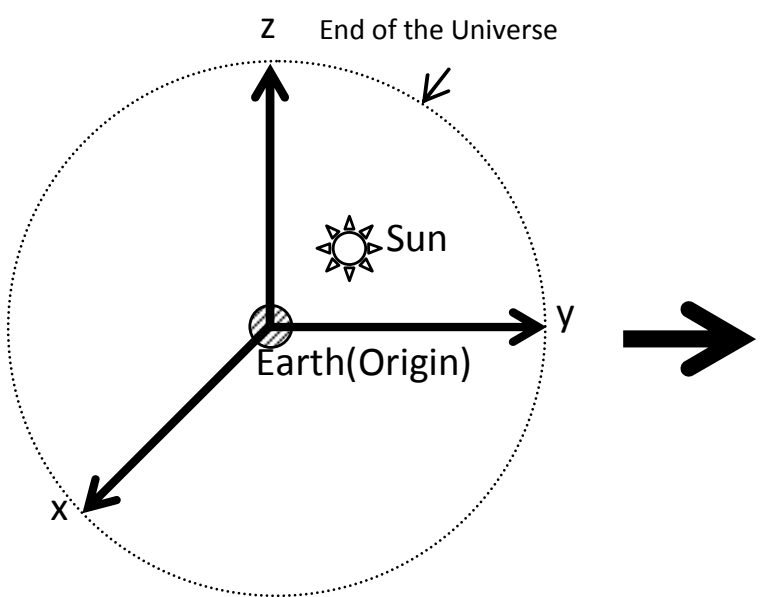

Figure 3

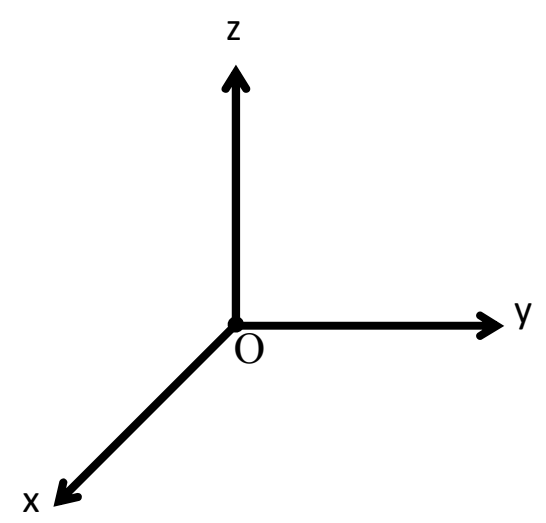

Figure 2

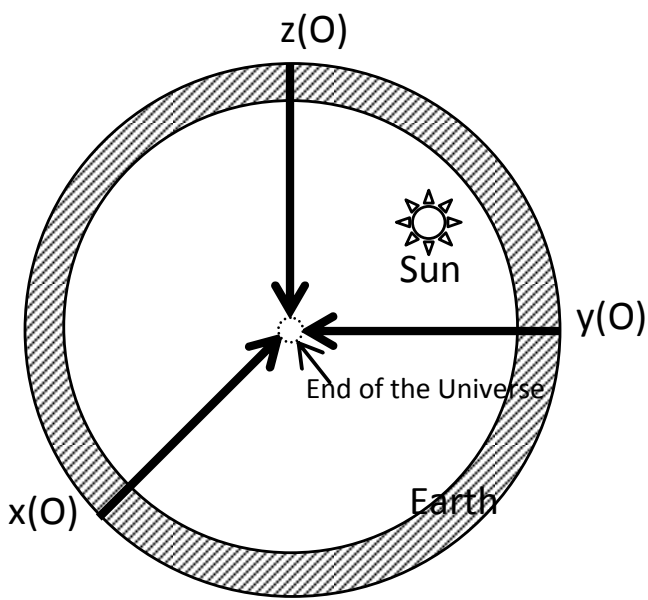

Figure 4

\section{Proof}

Now, let us think of the Poincaré conjecture that the compact 3-dimensional manifold is without boundaries by using the ordinary and reversed coordinates. First of all, using Figure 3 and 4 to facilitate grasping the process, let us glue these ordinary and reversed coordinates together at each end of the universes as shown in Figure 5. Thus, whole manifolds glued together are a closed $M^{3}$. 


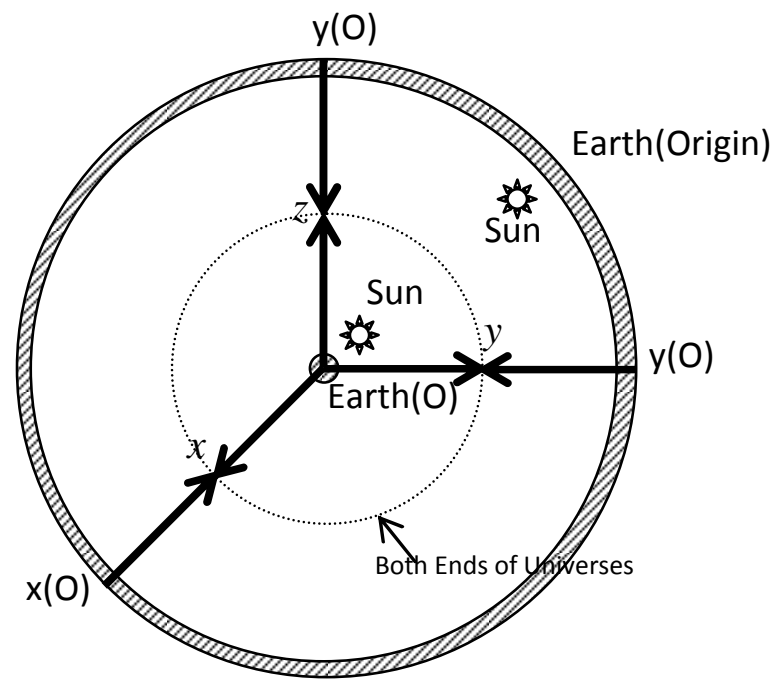

Figure 5

Secondarily, we verify if it is simply connected. Stretching up $\pi_{1}$ within the coordinates from the inner ordinary coordinate, it should shrink it down in the outer reversed coordinate. Thus $\pi_{1}$ will be gone. Now, let us pay attention to a fact: In the reversed coordinate, $\pi_{1}$ looks as if stretching up as it approaches to the reversed origin as shown in Figure 6. However, at the origin shaped a ring in the reversed coordinate, we can consider that $\pi_{1}\left(M^{3}\right)$ is contractible at the outmost. Because, it works inside out unlike in the ordinary coordinate as shown in Figure 7. On the other hand, they are topologically equivalent. Therefore, it is simply connected. Thus, the Poincaré conjecture on the 3-dimensional case is proved.

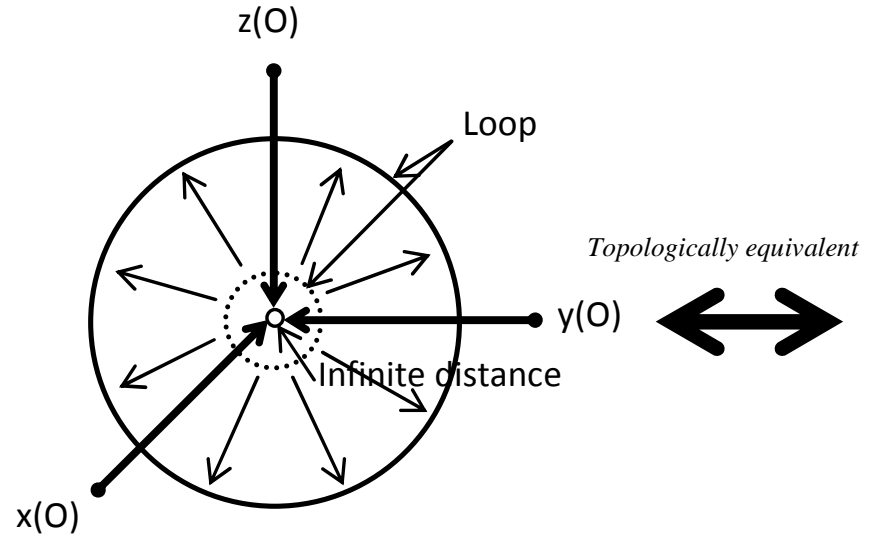

Figure 6

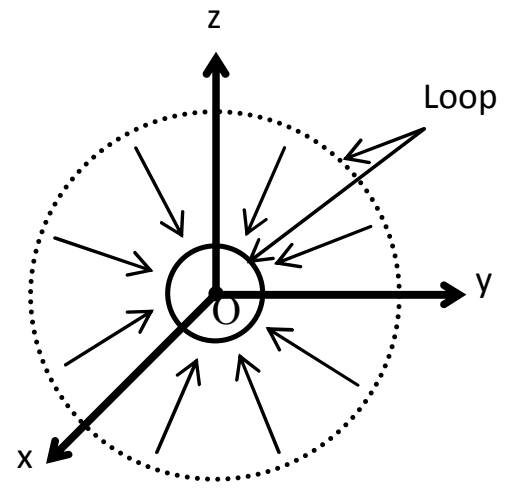

Figure 7 


\section{Acknowledgment}

I appreciate Frank Sapp with his support.

\section{References}

[1] Milnor, John (2004). "The Poincaré Conjecture 99 Years Later: A Progress Report" (PDF). Retrieved 2007-05-05.

[2] About the pdf file; http://www.math.sunysb.edu/ jack/PREPRINTS/poiproof.pdf 